\title{
TIME QUASI-PERIODIC TRAVELING GRAVITY WATER WAVES IN INFINITE DEPTH
}

\author{
ROBERTO FEOLA \\ University of Nantes, \\ e-mail: roberto.feola@univ-nantes.fr \\ FILIPPO GIULIANI \\ UPC, Barcelona, \\ e-mail: filippo.giuliani@upc.edu
}

\begin{abstract}
We present the recent result [8] concerning the existence of quasiperiodic in time traveling waves for the $2 d$ pure gravity water waves system in infinite depth. We provide the first existence result of quasi-periodic water waves solutions bifurcating from a completely resonant elliptic fixed point. The proof is based on a Nash-Moser scheme, Birkhoff normal form methods and pseudo-differential calculus techniques. We deal with the combined problems of small divisors and the fully-nonlinear nature of the equations.
\end{abstract}

\section{INTRODUCTION}

The aim of this note is to present the results obtained in [8] in which we prove the existence and the linear stability of small amplitude, quasi-periodic traveling solutions for the $2 d$ pure gravity water waves system in infinite depth. By a quasi-periodic traveling wave we mean a motion that, at the first orders of amplitude, is a superposition of an arbitrarily large number of periodic traveling waves with rationally independent frequencies. The irrationality of the frequencies of oscillations excludes the existence of a moving frame for which such motions are stationary. The existence of quasi-periodic in time water waves have been proved only recently, we refer to the papers [3], [1]. In these works the authors deal with cases in which the linear frequencies of oscillations are modulated by physical parameters, like the capillarity of the fluid or the depth of the ocean. In the pure gravity case with infinite depth the lack of such parameters allows the existence 
of infinite-dimensional subspaces which are invariant for the linearized problem and are filled by periodic in time solutions. We refer to this situation as a completely resonant case (see also [16]). Usually the complete resonance is referred as a stronger property of the linearized equation: all the solutions are periodic. Nevertheless the difficulties that we encounter are the same, indeed our solutions bifurcate from (possibly) periodic solutions. Then a careful nonlinear analysis is required to extract, directly from the system, some parameters that tune in an efficient way the frequencies of the expected solutions. This task is complicated by the presence of small divisors and the fully-nonlinear nature of the equations.

1.1. Formulation of the problem. We consider an incompressible and irrotational perfect fluid, under the action of gravity occupying, at time $t$, a two dimensional domain with infinite depth, periodic in the horizontal variable, given by

$$
\mathcal{D}_{\eta}:=\{(x, y) \in \mathbb{T} \times \mathbb{R} ;-\infty<y<\eta(t, x)\}, \quad \mathbb{T}:=\mathbb{R} /(2 \pi \mathbb{Z}) .
$$

The velocity field in the time dependent domain $\mathcal{D}_{\eta}$ is the gradient of a harmonic function $\Phi$, called the velocity potential. The time-evolution of the fluid is determined by a system of equations for the free surface $\eta(t, x)$, and the function $\psi(t, x)=\Phi(t, x, \eta(t, x))$ which is the restriction of the velocity potential $\Phi$ to the free interface. Given the shape $\eta(t, x)$ of the domain $\mathcal{D}_{\eta}$ and the Dirichlet value $\psi(t, x)$ of the velocity potential at the top boundary, one can recover $\Phi(t, x, y)$ as the unique solution of the elliptic problem

$$
\Delta \Phi=0 \text { in } \mathcal{D}_{\eta}, \quad \partial_{y} \Phi \rightarrow 0 \text { as } y \rightarrow-\infty, \quad \Phi=\psi \quad \text { on } \quad\{y=\eta(t, x)\} .
$$

According to Zakharov [19] and Craig-Sulem [5] the $(\eta, \psi)$ variables satisfy the gravity water waves system

$$
\left\{\begin{array}{l}
\partial_{t} \eta=G(\eta) \psi \\
\partial_{t} \psi=-g \eta-\frac{1}{2} \psi_{x}^{2}+\frac{1}{2} \frac{\left(\eta_{x} \psi_{x}+G(\eta) \psi\right)^{2}}{1+\eta_{x}^{2}}
\end{array}\right.
$$

where $G(\eta) \psi$ is the Dirichlet-Neumann operator

$$
G(\eta) \psi:=\left(\partial_{y} \Phi-\eta_{x} \partial_{x} \Phi\right)(t, x, \eta(t, x)) .
$$

Without loss of generality, we set the gravity constant to $g=1$.

It was first observed by Zakharov [19] that (1.2) is a Hamiltonian system with respect to the symplectic form $d \psi \wedge d \eta$ and it can be written as

$$
\partial_{t} \eta=\nabla_{\psi} H(\eta, \psi), \quad \partial_{t} \psi=-\nabla_{\eta} H(\eta, \psi),
$$

where $\nabla$ denotes the $L^{2}$-gradient, with Hamiltonian

$$
H(\eta, \psi):=\frac{1}{2} \int_{\mathbb{T}} \psi G(\eta) \psi d x+\frac{1}{2} \int_{\mathbb{T}} \eta^{2} d x
$$


given by the sum of the kinetic and potential energy of the fluid. The invariance of the system (1.2) in the $y$ and $x$ variable implies the existence of two prime integrals, respectively the "mass" $\int_{\mathbb{T}} \eta d x$ and the momentum

$$
\mathrm{M}:=\int_{\mathbb{T}} \eta_{x}(x) \psi(x) d x
$$

Since the ocean has infinite depth, if $\Phi$ solves (1.1), then $\Phi_{c}(x, y):=\Phi(x, y-c)$ solves the same problem in $\mathcal{D}_{\eta+c}$ assuming the Dirichlet datum $\psi$ at the free boundary $\eta+c$. Therefore $G(\eta+c)=G(\eta)$, for all $c \in \mathbb{R}$, and $\int_{\mathbb{T}} \nabla_{\eta} K d x=$ 0 where $K:=\frac{1}{2} \int_{\mathbb{T}} \psi G(\eta) \psi d x$ denotes the kinetic energy. Then $\widehat{\eta}_{0}(t):=$ $\frac{1}{2 \pi} \int_{\mathbb{T}} \eta(t, x) d x, \widehat{\psi}_{0}(t):=\frac{1}{2 \pi} \int_{\mathbb{T}} \psi(t, x) d x$ evolve according to the decoupled equations

$$
\partial_{t} \widehat{\eta}_{0}(t)=0, \quad \partial_{t} \widehat{\psi}_{0}(t)=-g \widehat{\eta}_{0}(t) .
$$

Hence we may restrict the study of the dynamics to the invariant subspace of $(\eta, \psi)$ such that

$$
\int_{\mathbb{T}} \eta d x=\int_{\mathbb{T}} \psi d x=0
$$

The Hamiltonian (1.4) is defined on the spaces

$$
(\eta, \psi) \in H_{0}^{s}(\mathbb{T} ; \mathbb{R}) \times H_{0}^{s}(\mathbb{T} ; \mathbb{R})
$$

where $H^{s}(\mathbb{T} ; \mathbb{R}), s \in \mathbb{R}$, denotes the Sobolev space of $2 \pi$-periodic functions of $x$, and $H_{0}^{s}(\mathbb{T} ; \mathbb{R})$ is the subspace of $H^{s}(\mathbb{T} ; \mathbb{R})$ of zero average functions.

Small amplitude solutions are close to the solutions of the linearized system of (1.2) at the equilibrium $(\eta, \psi)=(0,0)$, namely

$$
\partial_{t} \eta=G(0) \psi, \quad \partial_{t} \psi=-\eta
$$

where the Dirichlet-Neumann operator at the flat surface $\eta=0$ is the Fourier multiplier $G(0)=|D|$. Passing to the complex coordinates, the system (1.8) is equivalent to the following equation

$$
\partial_{t} u=-\mathrm{i}|D|^{\frac{1}{2}} u, \quad u=\frac{1}{\sqrt{2}}\left(|D|^{-\frac{1}{4}} \eta+\mathrm{i}|D|^{\frac{1}{4}} \psi\right) .
$$

The linear solutions have the form

$$
\begin{aligned}
& u(t, x)=\sum_{j \in \mathbb{Z} \backslash\{0\}} u_{j}(0) e^{-\mathrm{i} \sqrt{|j|} t+\mathrm{i} j x}, \\
& u_{j}(0):=\frac{1}{\sqrt{2}}\left(|j|^{-\frac{1}{4}} \eta_{j}(0)+\mathrm{i}|j|^{\frac{1}{4}} \psi_{j}(0)\right) .
\end{aligned}
$$

Such solutions can be either periodic, quasi-periodic or almost-periodic depending on the Fourier support. We remark that solutions initially Fourier supported on the infinite-dimensional invariant subspace $\left\{e^{\mathrm{i} n^{2} x}: n \in \mathbb{Z}\right\}$ are periodic in time, 
hence we say that the equation (1.9) is completely resonant. The frequency of oscillation of the $j$-th mode is $\sqrt{|j|}$ and we refer to the map

$$
j \rightarrow \sqrt{|j|}, \quad j \in \mathbb{Z} \backslash\{0\},
$$

as the dispersion law of (1.8). We note that the dispersion law is even in $j$, hence there are infinitely many multiple eigenvalues, and it grows sublinearly.

1.2. Main results. We define quasi-periodic traveling waves in the following way.

Definition 1. (Quasi-periodic traveling waves). ( $i)$ We say that a function

$$
(\eta(t, x), \psi(t, x)): \mathbb{R} \times \mathbb{T} \rightarrow \mathbb{R}^{2}
$$

is a quasi-periodic solution of (1.2) with irrational frequency vector $\omega \in \mathbb{R}^{\nu}$, if there is an embedding

$$
\begin{aligned}
\mathbb{T}^{\nu} & \rightarrow H_{0}^{1}(\mathbb{T} ; \mathbb{R}) \times H_{0}^{1}(\mathbb{T} ; \mathbb{R}) \\
\varphi & \mapsto U(\varphi, x):=(\tilde{\eta}(\varphi, x), \tilde{\psi}(\varphi, x))
\end{aligned}
$$

such that $(\eta(t, x), \psi(t, x))=U(\omega t, x)$ solves (1.2).

(ii) A quasi-periodic solution is traveling with velocity vector $\mathrm{v} \in \mathbb{Z}^{\nu}$ if there is a function $\widetilde{U}: \mathbb{T}^{\nu} \rightarrow \mathbb{R}^{2}$ such that

$$
(\eta(t, x), \psi(t, x))=U(\omega t, x)=\widetilde{U}(\omega t-\mathrm{v} x) .
$$

Remark 1.1. We remark that an embedding $U$ satisfies (1.12) if and only if $U(\varphi, x)$ solves the transport equation

$$
\mathrm{v} \cdot \partial_{\varphi} U+X_{\mathrm{M}}(U)=0, \quad X_{\mathrm{M}}(U)=\left(\tilde{\eta}_{x}, \tilde{\psi}_{x}\right),
$$

where $\mathrm{M}$ is the momentum Hamiltonian in (1.5).

We shall construct such solutions localized in Fourier space at $\nu$ distinct tangential sites $S:=S^{+} \cup S^{-}$

$$
S^{+}:=\left\{\bar{\jmath}_{1}, \ldots, \bar{\jmath}_{m}\right\} \subset \mathbb{N} \backslash\{0\}, \quad S^{-}:=\left\{\bar{\jmath}_{m+1}, \ldots, \bar{\jmath}_{\nu}\right\} \subset-\mathbb{N} \backslash\{0\},
$$

for some $1 \leq m \leq \nu$ and where

$$
k \neq-j, \quad \forall j \in S^{+}, \forall k \in S^{-} .
$$

The solutions of (1.8) that originate by exciting the tangential modes are superpositions of periodic traveling linear waves with velocity $\bar{j}_{i}$ and frequency $\sqrt{\left|\bar{\jmath}_{i}\right|}$. Such motions are quasi-periodic (or periodic) traveling waves of the form (1.12) with frequency vector

$$
\bar{\omega}:=\left(\sqrt{\left|\bar{\jmath}_{1}\right|}, \ldots, \sqrt{\left|\bar{\jmath}_{\nu}\right|}\right) \in \mathbb{R}^{\nu}
$$


and velocity vector

$$
\mathrm{v}:=\left(\bar{\jmath}_{1}, \ldots, \bar{\jmath}_{\nu}\right) \in \mathbb{Z}^{\nu}
$$

We construct small amplitude, quasi-periodic traveling waves solutions of (1.2) which are "close" to the linear ones, namely they will be of the form

$$
\begin{aligned}
& \eta(t, x)=\sum_{j \in S} \sqrt{2 \zeta_{j}}|j|^{\frac{1}{4}}\left(\cos \left(\omega_{j} t\right) \cos (j x)+\sin \left(\omega_{j} t\right) \sin (j x)\right)+o(\sqrt{|\zeta|}), \\
& \psi(t, x)=\sum_{j \in S} \sqrt{2 \zeta_{j}}|j|^{-\frac{1}{4}}\left(\cos \left(\omega_{j} t\right) \sin (j x)-\sin \left(\omega_{j} t\right) \cos (j x)\right)+o(\sqrt{|\zeta|}),
\end{aligned}
$$

where $\zeta=\left(\zeta_{j}\right)_{j \in S}$ with $\zeta_{j}>0, \omega=\bar{\omega}+O(|\zeta|)$ and $o(\sqrt{|\zeta|})$ is meant in the $H^{s}$-topology with $s$ large. The vectors $\left(\sqrt{\zeta_{j}}\right)_{j \in S}$ represent the amplitudes of the approximate solution from which we have the bifurcation. Our main result holds for a suitable choice of the tangental sites $\bar{j}_{i}$, that we prove to be generic. When we refer to a generic choice of the tangential sites we mean that the $\bar{\jmath}_{i}$ 's are chosen such that the vector $\left(\bar{\jmath}_{1}, \ldots, \bar{\jmath}_{\nu}\right)$ is not a zero of a certain non-trivial polynomial $\mathbb{C}^{\nu} \rightarrow \mathbb{C}$. We remark that such a choice is equivalent to the choice of the velocity vector $\mathrm{v}$ in 1.13 .

Denoting by $B(0, \varrho)$ the ball centered at the origin of $\mathbb{R}^{\nu}$ of radius $\varrho>0$, our result can be stated as follows.

Theorem 1. (Quasi-periodic traveling gravity waves). Let $\nu \geq 1$. For a generic choice of the velocity vector $\mathrm{v}$ as in (1.13) there exist $s \gg 1,0<\varrho \ll 1$ and a positive measure Cantor-like set $\mathfrak{A} \subseteq B(0, \varrho)$ such that the following holds. For any $\zeta \in \mathfrak{A}$, the equation (1.2) possesses a small amplitude, linearly stable, quasi-periodic solution $(\eta, \psi)(t, x ; \zeta)=U(\omega t, x ; \zeta)$ of the form (1.14) which is a traveling wave with velocity vector $\mathrm{v}, U(\varphi, x) \in H^{s}\left(\mathbb{T}^{\nu+1}, \mathbb{R}^{2}\right)$ and $\omega:=\omega(\zeta) \in$ $\mathbb{R}^{\nu}$ is a diophantine frequency vector. Moreover for $0<\varepsilon \leq \sqrt{\varrho}$, the set $\mathfrak{A}$ has asymptotically full relative measure in $\left[\varepsilon^{2}, 2 \varepsilon^{2}\right]^{\nu}$.

To the best of our knowledge the above theorem is the first existence result concerning quasi-periodic solutions of the water waves equations bifurcating from a completely resonant elliptic fixed point.

We mention Iooss-Plotnikov-Toland [16] and Iooss-Plotnikov [13] which proved for the completely resonant equation (1.2) the existence of standing periodic solutions.

Concerning the traveling waves, we cite Craig-Nicholls [4] which proved the existence of periodic solutions in the gravity-capillary case with space periodic boundary conditions. In absence of capillarity the existence of periodic traveling waves is a small divisor problem. This case has been treated by Iooss and Plotnikov in [14, 15]. 
The first results on the existence of quasi-periodic waves are quite recent and are due to Berti-Montalto [3] for the gravity-capillary case with infinite depth and by Baldi-Berti-Haus-Montalto [1] for the pure gravity case with finite depth for $2 \mathrm{~d}$ oceans. In both cases the existence of quasi-periodic solutions is provided for some asymptotically full-measure set of the parameters of the problem, respectively capillarity and depth (or equivalently wavelength).

As far as we know, all the previous results on periodic and quasi-periodic in time water waves take advantage from the presence of physical parameters and / or assumptions of parity conditions.

The purpose of the paper [8] is to address two natural questions:

(i) we work on a fixed equation for which the only possible parameters to modulate are the initial data of the solutions.

(ii) we look for a general class of quasi-periodic traveling waves which are free from restrictions of parity in the spatial and time variables.

Acknowledgements. The authors would like to thank the anonymous referee for very valuable and useful comments.

Roberto Feola has been supported of the Centre Henri Lebesgue ANR-11-LABX0020-01 and by ANR-15-CE40-0001-02 "BEKAM" of the Agence Nationale de la Recherche. Filippo Giuliani has received funding from the European Research Council (ERC) under the European Union's Horizon 2020 research and innovation programme under grant agreement No 757802.

\section{COMMENTS OF THE MAIN RESUlT}

Now we discuss the main issues and the novelties of the paper [8].

- The general form of the linear frequencies of oscillations for the water waves equations is the following

$$
\sqrt{|j| \tanh (\mathrm{h}|j|)\left(g+\kappa j^{2}\right)},
$$

where $\mathrm{h}$ and $\kappa$ are respectively the depth and the capillarity of the fluid. If $\mathrm{h}<\infty$ or $\kappa \neq 0$ such parameters may be used to impose non-resonance conditions that are needed for the search of quasi-periodic solutions (see for instance [1], [3], [18]). In our case $\mathrm{h}=\infty$ and $\kappa=0$, thus the linear frequencies of oscillations are $\sqrt{g|j|}$ and the elements of the infinite dimensional space $\operatorname{span}\left\{e^{\mathrm{i}|n| t} e^{\mathrm{i} n^{2} x}: n \in \mathbb{Z}\right\}$ are periodic solutions of the linearized problem at the origin (completely resonant case).

The physical parameter $g$ clearly does not modulate the frequencies, hence if we look for quasi-periodic solutions we need to extract parameters directly from the nonlinearities of the equation. We do that by means of Birkhoff normal form 
(BNF) techniques. In this way the bifurcation parameters are essentially the "initial data" or the amplitudes of an appropriate approximate solution from which the bifurcation occurs (see (1.14)). The choice of the Fourier support of such approximate solution plays a fundamental role in proving some non-degeneracy conditions. Roughly speaking, both the amplitudes $\sqrt{\zeta_{\bar{\jmath}_{i}}}$ and the tangential sites $\bar{\jmath}_{i}$ will be "parameters" of our problem.

- In performing BNF procedures we shall deal with resonances among linear frequencies. It is known that the pure gravity case in infinite depth has no 3-waves resonant interactions. On the other hand, there are many non-trivial 4-wave interactions, called Benjamin-Feir resonances (see (3.4)). We then exploit a fundamental property of the pure gravity waver waves Hamiltonian (1.4) in infinite depth: the formal integrability, up to order four, of the Birkhoff normal form. This has been proved in [7], [6], by showing explicit key algebraic cancellations occurring for the coefficients of the Hamiltonian.

- In order to show that the "initial data" of the expected solutions tune in an efficient way the frequencies we shall find the explicit expression of the first order corrections of the tangential frequencies and of the spectrum of the linearized operator in the normal directions. We use an identification argument of normal forms to detect them, in the spirit of [12], based on the presence of approximate constants of motion. Actually, even after this procedure, the Hamiltonian is still partially degenerate. Indeed it turns out that there are a finite number of eigenvalues which are still in resonance. We overcome this difficulty by passing to suitable rotating coordinates.

- We exhibit the existence of a wide class of traveling quasi-periodic solutions with no parity restrictions in time and space by using the Hamiltonian structure and the $x$-translation invariance of (1.2). It is well known that the water waves system (1.2) exhibits additional symmetries. For instance the vector field $X_{H}$ in (1.3) is

(i) reversible with respect to the involution

$$
S:\left(\begin{array}{c}
\eta(x) \\
\psi(x)
\end{array}\right) \mapsto\left(\begin{array}{c}
\eta(-x) \\
-\psi(-x)
\end{array}\right)
$$

i.e. it satisfies $X_{H} \circ S=-S \circ X_{H}$;

(ii) even-to-even, i.e. maps $x$-even functions into $x$-even functions.

In several papers (for instance [1], [3], [16], [18]) such symmetries are adopted to remove degeneracies due to translation invariance in $x$ and $t$. Since we do not look for solutions in the subspace of reversible functions, namely

$$
\{(\eta, \psi) \text { such that }(\eta, \psi)(t, x)=(\eta,-\psi)(-t,-x)=S(\eta, \psi)(-t, x)\},
$$


the existence of a solution $U(t, x)$ of (1.2) implies the existence of a possibly different solution $S U(-t, x)$.

- The vector field in (1.2) is a singular perturbation of the linearized system at the origin (1.8) since the nonlinearity contains derivatives of the first order, while (1.9) contains only derivatives of order $1 / 2$. We remark that this is not the case when the capillarity $\kappa \neq 0$.

- The dispersion law (1.10) is sub-linear. This is a major difference in developing KAM theory for equations with super-linear dispersion law, such as in the gravitycapillary case. Indeed a weaker dispersion law implies bad separation properties of the eigenvalues. The main issue concerns the verification of non-resonance conditions between the tangential frequencies and the difference of the normal ones, called "second order Melnikov conditions".

\section{IDEAS OF THE PROOF}

Here we summarize the steps of the proof of Theorem 1 highlighting the key ingredients.

Nash-Moser theorem of hypothetical conjugation. We formulate the problem of finding quasi-periodic solutions as the problem of the search for zeros of a nonlinear functional equation

$$
\mathcal{F}(\omega, U(\omega ; \varphi, x))=0
$$

where $U(\omega ; \varphi, x)$ is a smooth embedding of a $\nu$-dimensional torus supporting a quasi-periodic motion with frequency vector $\omega \in \mathbb{R}^{\nu}$ (see (1.11)). We implement a Nash-Moser scheme which provides the zeros of such a functional as limit of a sequence $\left(U_{n}\right)_{n \geq 0}$ of approximate solutions

$$
U_{n+1}:=U_{n}-\Pi_{n}\left(d \mathcal{F}\left(\omega ; U_{n}\right)\right)^{-1} \Pi_{n} \mathcal{F}\left(U_{n}\right),
$$

where $\Pi_{n}$ is a smoothing operator, that converges in some very regular Sobolev space. The main issues concern the invertibility of the linearized operator (with suitable estimates) in a neighborhood of the equilibrium and the search for a good approximate solution $U_{0}$ that initializes the scheme. This task is doable only for appropriate choices of the parameter frequency $\omega$. Then we prove that the set of such good frequencies has positive Lebesgue measure.

Bifurcation analysis. We find the first nonlinear approximation $U_{0}$ as a solution of a "simplified" Hamiltonian system whose dynamics is close to (1.4), at least for a certain time range. Since we work in a neighborhood of an elliptic fixed point, this Hamiltonian can be obtained through a weak version of the Birkhoff normal form method (WBNF), where the adjective weak refer to the fact that we just "partially" normalize the Hamiltonian (1.4). More precisely we construct a map, which is close to the identity up to a finite rank operator, such that the Hamiltonian 
$H$ in (1.4), in the complex coordinates (1.9), assumes the form $H_{\text {Birk }}+R$, where $R$ can be considered as a small remainder in a neighborhood of the origin,

$$
\begin{aligned}
& H_{\text {Birk }}=H_{\mathbb{C}}^{(2)}+\mathcal{H}_{\mathbb{C}}^{(4,0)}+\mathcal{H}_{\mathbb{C}}^{(\geq 6,0)}, \quad H_{\mathbb{C}}^{(2)}=\sum_{j \in \mathbb{Z}} \sqrt{|j|}\left|u_{k}\right|^{2} \\
& \mathcal{H}_{\mathbb{C}}^{(4,0)}:=\frac{1}{4 \pi} \sum_{k \in S}|k|^{3}\left|u_{k}\right|^{4}+\frac{1}{\pi} \sum_{\substack{k_{1}, k_{2} \in S, \operatorname{sign}\left(k_{1}\right)=\operatorname{sign}\left(k_{2}\right) \\
\left|k_{2}\right|<\left|k_{1}\right|}}\left|k_{1}\right|\left|k_{2}\right|^{2}\left|u_{k_{1}}\right|^{2}\left|u_{k_{2}}\right|^{2}
\end{aligned}
$$

and $\mathcal{H}_{\mathbb{C}}^{(\geq 6,0)}$ is some homogeneous integrable Hamiltonian, in the sense that it depends only on the actions $\left|u_{k}\right|^{2}$, of degree 6 . We remark that $\mathcal{H}_{\mathbb{C}}^{(4,0)}, \mathcal{H}_{\mathbb{C}}^{(\geq 6,0)}$ are supported on the tangential set $S$, while $R$ contains monomials supported at least on one mode out of $S$. The finite dimensional subspace $U_{S}:=\left\{u_{k}=0 \forall k \notin\right.$ $S\}$ turns out to be invariant for $H_{\text {Birk }}$. We introduce the following action-angle variables on $U_{S}$

$$
u_{k}=\sqrt{I_{k}} e^{-\mathrm{i} \theta_{k}} \quad k \in S, \quad u_{k}=z_{k} \quad k \notin S .
$$

In these coordinates $\mathcal{H}_{\mathbb{C}}^{(4,0)}=\frac{1}{2} \mathbb{A} I \cdot I$, where $\mathbb{A}$ is a symmetric $\nu \times \nu$ matrix. We prove that the Hamiltonian restricted to $U_{S}$ is integrable and non-degenerate in the sense that the frequency-to-amplitude map

$$
\omega(I)=\bar{\omega}+\mathbb{A} I+O\left(I^{2}\right)
$$

is a local diffeomorphism. Then we can select $U_{0}$ among the tori $\{I=\zeta\}$, where $\zeta$ is some fixed vector with positive components. In order to work in a neighborhood of these tori is convenient to rescale the unperturbed actions $\zeta \rightarrow \varepsilon^{2} \zeta$ with a small parameter $\varepsilon>0$. The non-degeneracy of the frequency-amplitude map allows to impose non-resonance conditions such as

$$
|\omega \cdot \ell| \geq \frac{\gamma}{\langle\ell\rangle^{\tau}} \quad \forall \ell \in \mathbb{Z}^{\nu} \quad \tau>\nu-1
$$

where

$$
\gamma=o\left(\varepsilon^{2}\right) .
$$

Notice that the condition (3.2) could be obtained just by choosing generically the tangential sites in $S$. However, in order to impose higher order non-resonance conditions required by the Nash-Moser scheme, we truly need the non-degeneracy of the frequency-to-amplitude map. We remark that the dependence of $\gamma$ in 3.3. respect to $\varepsilon$ is a peculiarity of resonant cases, indeed this is due to the closeness of $\omega$ to the resonant vector $\bar{\omega}$. For non-resonant cases $\gamma$ can be considered as a small number independent of $\varepsilon$. In the search for small amplitude solutions for resonant equations this fact produces several difficulties in the bifurcation analysis. For instance, in our case, we have to implement several steps in the Birkhoff normal 
form procedures that we perform along the Nash-Moser iteration. In the normal form analysis we have to deal with waves resonant interactions such as

$$
\sum_{i=1}^{n} \sigma_{i} j_{i}=0, \quad \sum_{i=1}^{n} \sigma_{i} \sqrt{\left|j_{i}\right|}=0, \quad \sigma_{i}= \pm, \quad i=1, \ldots, n .
$$

We say that a $n$-tuple $\left(j_{1}, \ldots, j_{n}\right)$ is a trivial resonance if $n$ is even, $\sigma_{i}=-\sigma_{i+1}$, $i=1, \ldots, n-1$ (up to permutations), and the $n$-tuple has the form $(j, j, k, k, \ldots)$. It is easy to note that monomials $u_{j_{1}} \ldots u_{j_{n}}$ supported on trivial resonances are integrable, meaning that depend only on the actions $\left|u_{j}\right|^{2}$. For $n=4$ there are infinitely many non-trivial resonances, called Benjamin-Feir resonances, which consist in the two parameter family of solutions

$$
\bigcup_{\lambda \in \mathbb{Z} \backslash\{0\}, b \in \mathbb{N}}\left\{j_{1}=-\lambda b^{2}, j_{2}=\lambda(b+1)^{2}, j_{3}=\lambda\left(b^{2}+b+1\right)^{2}, j_{4}=\lambda(b+1)^{2} b^{2}\right\},
$$

with $\sigma_{1}=\sigma_{3}=-\sigma_{2}=-\sigma_{4}$. In [7], [6] it has been proved that the coefficients of the normalized Hamiltonian (obtained by a full Birkhoff normal form procedure) at order four of the monomials corresponding to the Benjamin-Feir resonances vanish. By using suitable algebraic arguments, we actually prove that such cancellations of [7], [6] occur also preforming the weak version of BNF which involves only finitely many tangential sites. In this way we conclude the integrability of the weak BNF Hamiltonian at degree 4 and we obtain its explicit formula (3.1). In order to deal with higher order resonances we use a genericity argument.

Normal form identification. The frequency of the expected quasi-periodic solutions are small corrections of the linear frequencies of oscillations $\bar{\omega}$. Then the first order corrections are fundamental to impose the non-resonance conditions. Usually such corrections are obtained through a Birkhoff normal form procedure that normalizes the full Hamiltonian at order four, i.e. that normalizes all the monomials of order four. This procedure has been implemented in [6], [7] at formal level, where the integrability of the normal form is provided by explicit computations of the coefficients of the Hamiltonian. It is natural to expect that the first order corrections can be computed from the normal form obtained in this way. However, due to the quasi-linear nature of the equations, it is not trivial to provide rigorous bounds on the changes of coordinates that allow to construct a reducible (up to certain order) normal form around the torus. Thus we follow a different strategy.

To explain it we first compare different Birkhoff normal form approaches whose aim is to normalize different type of monomials in the Hamiltonian.

- Full BNF: all the monomials of degree three and four are normalized; 
- Weak + Linear BNF: it is divided into two steps: (i) normalization of all the cubic and quartic terms with at most one wave number outside the set of tangential sites $S$ (weak); (ii) normalization of all the cubic and quartic terms with exactly two wave numbers outside the set $S$ (linear). We remark that both procedures are not convergent. We follow a strategy close to the weak+linear BNF and we prove that the corrections found with our approach coincide with the ones we expect. We do not explicitly compute such corrections but we prove a uniqueness result on the normal form which implies an a posteriori identification. In non resonant cases this kind of results are provided by classical arguments, see for instance [17]. For the resonant equation (1.2) the main difficulty is due to the presence of non trivial resonances at order four, the Benjamin-Feir resonances, that a priori do not guarantee the integrability nor the uniqueness of the normal form around the torus. To overcome this problem we would like to obtain the same cancellations on the resonant coefficients of the Hamiltonian obtained in [7]. We do that by exploiting the approximate constants of motion of the system (in the spirit of [10]).

The main idea is the following: given two Hamiltonians $H=H^{(2)}+O\left(u^{3}\right)$ and $K=K^{(2)}+O\left(u^{3}\right)$ that commute (up to some order) there exists a change of coordinates $\Psi$ that puts simultaneously the Hamiltonians in normal form (up to the same order) namely

$$
H \circ \Psi=H^{(2)}+Z+R, \quad K \circ \Psi=K^{(2)}+W+Q,
$$

where the normalized terms $Z, W$ are such that $\left\{H^{(2)}, Z\right\}=0,\left\{K^{(2)}, W\right\}=0$ and $R, Q$ have higher order of homogeneity. Moreover

$$
\left\{Z, K^{(2)}\right\}=\left\{H^{(2)}, W\right\}=0 \text {. }
$$

Hence the normalized Hamiltonian $Z$ (as well as $W$ ) is Fourier supported on the common resonances of the adjoint actions of $H^{(2)}$ and $K^{(2)}$. This implies that the coefficients of $Z$ related to non common resonances are automatically zero.

We exploit the formal integrability at order four to construct an approximate constant of motion $K$ for the Hamiltonian (1.4) with the following property: the adjoint action of $K^{(2)}$ possesses only trivial resonances. This allows to recover a posteriori the cancellations of the coefficients of the non-integrable resonant monomials of the normal form and provide the uniqueness argument.

Invertibility of the linearized operator. In a suitable set of coordinates the linear dynamics of the tangential (to the torus) variables is decoupled by the dynamics of the normal ones (we follow the Berti-Bolle method [2]). The main difficulty is to invert the linearized operator in the normal directions $\mathcal{L}_{\omega}=\mathcal{L}_{\omega}\left(U_{n}\right)$ at each step of the Nash-Moser scheme. We have that $\mathcal{L}_{\omega}$ is given, up to finite rank operators, 
by

$$
\omega \cdot \partial_{\varphi}+\left(\begin{array}{cc}
\partial_{x} V+G(\eta) B & -G(\eta) \\
\left(1+B V_{x}\right)+B G(\eta) B & V \partial_{x}-B G(\eta)
\end{array}\right)
$$

where

$$
\begin{aligned}
& V=V(\eta, \psi):=\left(\partial_{x} \Phi\right)(x, \eta(x))=\psi_{x}-\eta_{x} B, \\
& B=B(\eta, \psi):=\left(\partial_{y} \Phi\right)(x, \eta(x))=\frac{G(\eta) \psi+\eta_{x} \psi_{x}}{1+\eta_{x}^{2}} .
\end{aligned}
$$

We observe that $\mathcal{L}_{\omega}$ is a pseudo differential operator of order one. Our aim is to obtain the invertibility of $\mathcal{L}_{\omega}$ and to provide suitable tame estimates on the inverse. We do that by means of a reducibility argument, namely we find a quasiperiodically time dependent change of coordinates $\mathcal{T}=\mathcal{T}(\omega t)$ that conjugates $\mathcal{L}_{\omega}$ to a diagonal (in the Fourier basis) operator. This strategy consists into two main steps:

(a) a pseudo differential reduction in decreasing order of $\mathcal{L}_{\omega}$ which conjugates the linearized operator to a pseudo differential one with constant coefficients up to a bounded remainder;

(b) a reduction of bounded operators and a KAM scheme which complete the diagonalization;

The main new issues are the following:

(I) by the complete resonance the diophantine constants $\gamma$ in (3.8) and $\eta$ in (3.9) are small with the size of the amplitudes (see for instance (3.3)). This implies that many terms in $\mathcal{L}_{\omega}$ are not perturbative for the KAM scheme (b), which requires a smallness condition like $\varepsilon^{a} \gamma^{-1} \ll 1$ for some $a>0$.

(II) We consider the linearization on a quasi-periodic traveling embedding $U(\varphi, x)$ without any assumption on the parity of $\varphi$ and $x$. Usually such conditions provide some algebraic cancellations in performing steps $(a)$ (b) and reduce the multiplicity of the eigenvalues simplifying the proof.

We deal with (I) by splitting each step of the reducibility into 2 parts. In the first part we treat the non perturbative terms of $\mathcal{L}_{\omega}$ by using algebraic arguments to deal with resonances between the linear frequencies of oscillation. We refer to these steps as preliminary steps and linear Birkhoff normal form steps when they are performed in the procedures described in items $(a)$ and $(b)$ respectively. After the procedures $(a)$ and $(b)$ we obtain a map $\mathcal{T}=\mathcal{T}(\omega t)$ such that

$$
\mathcal{T} \mathcal{L}_{\omega} \mathcal{T}^{-1}=\omega \cdot \partial_{\varphi}+D, \quad D=\operatorname{diag}_{j \in \mathbb{Z}}\left(d_{j}\right)
$$

where

$$
d_{j}:=\mathfrak{m}_{1} j+\left(1+\mathfrak{m}_{\frac{1}{2}}\right) \sqrt{|j|}+\mathfrak{m}_{0} \operatorname{sign}(j)+r_{j}
$$


with $\mathfrak{m}_{1 / 2}, \mathfrak{m}_{0}, r_{j}$ depending on $\omega$ and satisfying

$$
\begin{gathered}
\mathfrak{m}_{1}=\varepsilon^{2} m_{1}+O\left(\varepsilon^{4}\right), \quad m_{1}:=\frac{1}{\pi} \sum_{n \in S} n|n| \zeta_{n}, \\
\left(\sup _{j}|j|^{-1 / 2}\left|r_{j}\right|+\left|\mathfrak{m}_{1 / 2}\right|+\left|\mathfrak{m}_{0}\right|\right) \gamma^{-1} \ll 1 .
\end{gathered}
$$

We point out that the first order corrections $O\left(\varepsilon^{2}\right)$ of the $d_{j}$ 's are fundamental to impose the non-resonance conditions since the linear equation (1.9) is completely resonant. Usually such corrections comes from the normalization of the Hamiltonian terms depending on 2 normal variables. We remark that the weak BNF leaves untouched these terms. Then we use an identification argument to prove that this normalization is obtained through the preliminary steps and the linear Birkhoff normal form aforementioned.

Concerning (II), we have to deeply exploit the Hamiltonian structure and the conservation of momentum. More precisely the map $\mathcal{T}$ constructed in steps $(a)$, (b) is obtained as a composition of several symplectic maps that preserve the subspace of traveling embeddings $U(\varphi, x)=\widetilde{U}(\varphi-\mathrm{v} x)$. This allows to obtain algebraic cancellations that provide the diagonalization in decreasing order of the operator $\mathcal{L}_{\omega}$.

Now we describe an important step of the pseudo differential reduction of $\mathcal{L}_{\omega}$, namely the reduction of the highest order term where we have to deal with the singular perturbation problem. For simplicity we consider the following model example

$$
\omega \cdot \partial_{\varphi}+V(\varphi, x) \partial_{x}+(1+a(\varphi, x))|D|^{1 / 2} .
$$

The operator $\mathcal{L}_{\omega}$ assumes a similar form after a block-diagonalization and passing to "good unknown" variables. Our aim is to find a change of coordinates that conjugates (3.6) to

$$
\omega \cdot \partial_{\varphi}+\mathfrak{m}_{1} \partial_{x}+\left(1+a_{+}(\varphi, x)\right)|D|^{1 / 2}+\ldots
$$

where ... denotes lower order pseudo differential operators and $\mathfrak{m}_{1}$ is in (3.5). Actually this problem is equivalent to straighten the degenerate vector field $\omega$. $\frac{\partial}{\partial \varphi}+V(\varphi, x) \frac{\partial}{\partial x}$ on the $\nu+1$-dimensional torus. A priori, due to the degeneracy $V=O(\varepsilon)$, we cannot eliminate the $x$-dependence of the vector field by perturbative arguments. By using the conservation of momentum we can reduce one degree of freedom and study the following non-degenerate vector field

$$
(\omega-\tilde{V}(\Theta) \mathrm{v}) \cdot \frac{\partial}{\partial \Theta}, \quad \Theta \in \mathbb{T}^{\nu},
$$

where $\widetilde{V}(\varphi-\mathrm{v} x)=V(\varphi, x)$. Then we can apply a result of straightening of weakly perturbed constant vector fields on tori given in [9]. We remark that $\mathfrak{m}_{1} \approx$ 
$(2 \pi)^{-\nu} \int_{\mathbb{T}^{\nu}} \widetilde{V}(\Theta) d \Theta$

When we conjugate the operator (3.6) into (3.7) we need to ensure that the pseudo differential structure is preserved and we need to provide suitable estimates for the lower order terms. We do that by using a quantitative Egorov theorem developed in [11].

(iv) Non-resonance conditions. Since the dispersion law is sub-linear, at each step of items $(a)$ and $(b)$ small divisors problems arise. We discuss the nonresonance conditions that we shall require on the eigenvalues. In order to deal with the operator in (3.6) we shall impose the following "zero order Melnikov conditions":

$$
\left|\left(\omega-\mathfrak{m}_{1} \mathrm{v}\right) \cdot \ell\right| \geq \gamma\langle\ell\rangle^{-\tau}, \quad \forall \ell \in \mathbb{Z}^{\nu} \backslash\{0\} .
$$

In the KAM reducibility scheme in item $(b)$ we shall impose suitable lower bounds on the function

$$
\psi_{\ell, j, k}=\omega \cdot \ell+d_{j}-d_{k}
$$

with $\ell \in \mathbb{Z}^{\nu}, j, k \in \mathbb{Z} \backslash\{0\}$. These conditions are called "second order Melnikov conditions" and are of the form

$$
\left|\psi_{\ell, j, k}\right| \geq \frac{\eta}{\langle\ell\rangle^{\tau}}, \quad \ell \in \mathbb{Z}^{\nu}, \quad j, k \in \mathbb{Z} \backslash\{0\}, \quad(\ell, j, k) \neq(0, j, j),
$$

where $\eta, \tau>0$ are some constants to be fixed. We prove that (3.9) hold with

$$
\eta:=\gamma^{3} \ll \gamma,
$$

where $\gamma$ is the diophantine constant in (3.8). Actually we show that the complementary of the set of frequencies $\omega$ such that (3.9) hold has measure going to zero as $\varepsilon \rightarrow 0$. The crucial problem is the summability in the indexes $\ell, j, k$. Since in this case the linear frequencies grow at infinity in sublinear way, the difference $\sqrt{|j|}-\sqrt{|k|}$ accumulates everywhere in $\mathbb{R}$. This means that for any fixed $\ell$ there are infinitely many indexes $j, k$ to be taken into account. The key idea that we use is the following. By the conservation of momentum we need to impose the conditions (3.9) only for $(\ell, j, k)$ satisfying

$$
\mathrm{v} \cdot \ell+j-k=0,
$$

where $\mathrm{v}$ is the velocity vector given in (1.13). This allows us to show that, if $|j|,|k|$ are much larger than $|\ell|$, then the conditions (3.9) are implied by the (3.8) and then we are left to control the small divisors only for finitely many indexes $j, k$.

An alternative approach would be to prove Melnikov conditions by setting

$$
\eta:=\frac{\gamma}{\langle j\rangle^{\mathrm{d}}\langle k\rangle^{\mathrm{d}}}, \quad \mathrm{d}>1
$$


This choice would allow to prove that "many" frequencies $\omega$ satisfy the (3.9), but with the disadvantage of having small divisors that create a loss of space derivatives.

The class of non perturbative terms in the case $\gamma^{3}=\eta$ is larger respect to the choice $\gamma=\eta$, however such terms are compactly time-Fourier supported $(|\ell| \leq C$ for some constant $C>0$ ). In [8], in order to deal with such non perturbative terms, we adopt the following strategy: if at least one between $|j|,|k|$ is large enough then there are no small divisors; otherwise we choose to impose the second order resonance Melnikov conditions with $\eta$ as in (3.11). Actually this does not create any loss of derivatives since $|j|,|k|$ are taken into a ball with finite radius. We deal with the perturbative terms by imposing conditions (3.9) with $\eta$ as in 3.10).

\section{REFERENCES}

[1] P. Baldi, M. Berti, E. Haus, R. Montalto, Time quasi-periodic gravity water waves in finite depth, Invent. math. 214, 739-911, (2018).

[2] M. Berti, P. Bolle, A Nash-Moser approach to KAM theory, Fields Institute Communications, volume 75 "Hamiltonian PDEs and Applications", 255-284, (2014).

[3] M. Berti, R. Montalto, KAM for gravity capillary water waves, Memoires of AMS, Memo 891, vol. 263 (2020).

[4] W. Craig, D. Nicholls, Travelling two and three dimensional capillary gravity water waves, SIAM J. Math. Anal., 32(2):323-359, (2000).

[5] W. Craig, C. Sulem, Numerical simulation of gravity waves, J. Comput. Phys., 108(1):73-83, (1993).

[6] W. Craig, P. Worfolk, An integrable normal form for water waves in infinite depth, Phys. D, 84(3-4):513-531, (1995).

[7] A. I. Dyachenko, V. E. Zakharov, Is free-surface hydrodynamics an integrable system? Physics Letters A, 190(2):144-148, (1994).

[8] R. Feola, F. Giuliani, Quasi-periodic Traveling Waves on an Infinitely Deep Perfect Fluid Under Gravity, preprint, arXiv:2005.08280 (2020).

[9] R. Feola, F. Giuliani, R. Montalto, M. Procesi, Reducibility of first order linear operators on tori via Moser's theorem, Journal of Functional Analysis 276(3):932-970 (2019). DOI: https://doi.org/10.1016/j.jfa.2018.10.009.

[10] R. Feola, F. Giuliani, S. Pasquali, On the integrability of Degasperis-Procesi equation: control of the Sobolev norms and Birkhoff resonances, Journal of Differential Equations 266(6):3390 3437, (2019). DOI: https://doi.org/10.1016/j.jde.2018.09.003.

[11] R. Feola, F. Giuliani, M. Procesi, Reducibility for a class of weakly dispersive linear operators arising from the Degasperis Procesi equation, Dynamics of Partial Differential Equations, 16(1): 25-94 (2019). DOI: http://dx.doi.org/10.4310/DPDE.2019.v16.n1.a2.

[12] R. Feola, F. Giuliani, M. Procesi, Reducibile KAM tori for the Degasperis Procesi equation, Comm. Math. Phys. (2020), DOI: 10.1007/s00220-020-03788-z.

[13] G. Iooss, P. Plotnikov, Multimodal standing gravity waves: a completely resonant system, J. math. fluid mech. 7, S110-S126, (2005). 
[14] G. Iooss, P. Plotnikov, Small divisor problem in the theory of three-dimensional water gravity waves, Mem. Amer. Math. Soc., 200(940), (2009).

[15] G. Iooss, P. Plotnikov, Asymmetrical tridimensional traveling gravity waves, Arch. Rat. Mech. Anal., 200(3):789-880, (2011).

[16] G. Iooss, P. Plotnikov, J. Toland, Standing waves on an infinitely deep perfect fluid under gravity, Arch. Ration. Mech. Anal., 177(3):367-478, 2005.

[17] T. Kappeler, J. Pöschel, KAM and KdV, Springer (2003).

[18] P. Plotnikov, J. Toland, Nash-Moser theory for standing water waves. Arch. Ration. Mech. Anal., 159(1):1-83, 2001.

[19] V.E. Zakharov, Stability of periodic waves of finite amplitude on the surface of a deep fluid, J Appl Mech Tech Phys 9:190-194, (1968). 